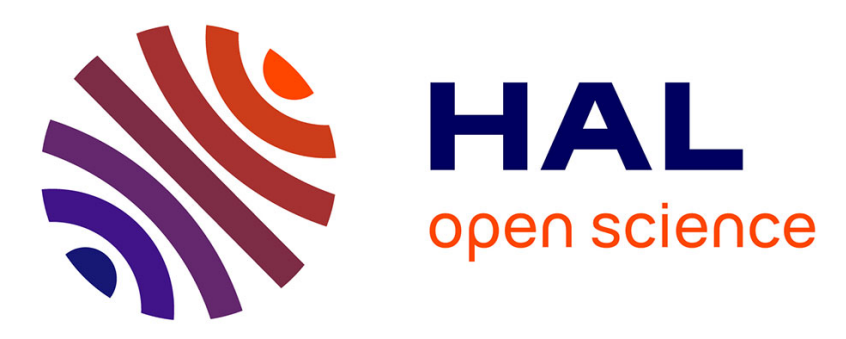

\title{
docExtractor: An off-the-shelf historical document element extraction
}

\author{
Tom Monnier, Mathieu Aubry
}

\section{To cite this version:}

Tom Monnier, Mathieu Aubry. docExtractor: An off-the-shelf historical document element extraction. 2020 17th International Conference on Frontiers in Handwriting Recognition (ICFHR), Sep 2020, Dortmund, France. pp.91-96, 10.1109/ICFHR2020.2020.00027 . hal-03131070

\author{
HAL Id: hal-03131070 \\ https://hal.science/hal-03131070
}

Submitted on 4 Feb 2021

HAL is a multi-disciplinary open access archive for the deposit and dissemination of scientific research documents, whether they are published or not. The documents may come from teaching and research institutions in France or abroad, or from public or private research centers.
L'archive ouverte pluridisciplinaire HAL, est destinée au dépôt et à la diffusion de documents scientifiques de niveau recherche, publiés ou non, émanant des établissements d'enseignement et de recherche français ou étrangers, des laboratoires publics ou privés. 


\title{
docExtractor: An off-the-shelf historical document element extraction
}

\author{
Tom Monnier and Mathieu Aubry \\ LIGM, École des Ponts, Univ Gustave Eiffel, CNRS, Marne-la-vallée, France \\ \{tom.monnier, mathieu.aubry\}@enpc.fr
}

\begin{abstract}
We present docExtractor, a generic approach for extracting visual elements such as text lines or illustrations from historical documents without requiring any real data annotation. We demonstrate it provides high-quality performances as an offthe-shelf system across a wide variety of datasets and leads to results on par with state-of-the-art when fine-tuned. We argue that the performance obtained without fine-tuning on a specific dataset is critical for applications, in particular in digital humanities, and that the line-level page segmentation we address is the most relevant for a general purpose element extraction engine. We rely on a fast generator of rich synthetic documents and design a fully convolutional network, which we show to generalize better than a detection-based approach. Furthermore, we introduce a new public dataset dubbed IlluHisDoc dedicated to the fine evaluation of illustration segmentation in historical documents.
\end{abstract}

Index Terms - deep learning, document layout analysis, historical document, page segmentation, text line detection, synthetic data

\section{INTRODUCTION}

In the context of a rising interest in digital humanities, the need for easy-to-use and efficient tools to automatically analyse document images has dramatically increased. Yet, document analysis is usually broken down into multiple subproblems depending on the precise task (paragraph-level page segmentation, text line detection, photograph or illumination extraction, etc.) and the specific type of document (modern or historical, printed or handwritten, its language, its condition, etc.), each often treated independently and requiring a specific set of training data and annotations. It is thus difficult for nonspecialists to find their way to the suited solution and universal engines that can tackle multiple tasks across various types of documents would be highly beneficial.

With the rise of deep learning, impressive improvements have been made in the document analysis domain. Neuralbased methods not only have set new state-of-the-art in most of document layout analysis tasks, but also enabled the development of powerful generic solutions that can tackle multiple analysis tasks with a same core method. Nonetheless, each task specific solution can hardly be used off-the-shelf as it always requires a dedicated training phase, involving a considerable amount of annotations and some expertise.

In this work, we tackle the problem of document element extraction as a unified line-level page segmentation task. We present a fast and scalable synthetic document generation engine that produces a wide diversity of documents with finegrained ground truth. We show that a fully convolutional network trained on resulting dataset called SynDoc (i) is a powerful off-the-shelf system with remarkable performances across multiple layout analysis tasks and (ii) leads to stateof-the-art results when fine-tuned with real data. In a detailed ablation study, we demonstrate that our new data generation process as well as our proposed network architecture are key components for these results. To better evaluate generalization, we also introduce a new public test dataset dubbed IlluHisDoc and dedicated to the evaluation of illustration segmentation methods for historical documents.

Synthetic generation pipeline, network implementation and IlluHisDoc dataset are all available at our project webpage: http://imagine.enpc.fr/ monniert/docExtractor/.

\section{RELATED WORK}

Page segmentation. Also called document layout analysis, page segmentation is an active research area with numerous competitions [1]-[4] and datasets [5]-[8]. They usually consider many semantic categories (e.g., caption, paragraph, title) and split text regions at paragraph level. To perform text recognition, text line detection needs to be performed with dedicated methods such as described in the next paragraph. We argue that for many practical applications on historical documents in which layout is often simple, important elements are illustrations and text lines. In contrast to prior work, we thus target segmenting illustrations and text lines jointly, a problem we refer to as line-level page segmentation.

Text line detection. While text line detection in modern printed documents is considered as a solved problem, it remains challenging for historical documents [2], [9]-[11]. In most recent competitions [9], [11] the task is actually to detect text baselines, which represent a compromise between annotation cost and descriptive power. We use instead the $\mathrm{x}$ height representation [12] which not only enables to infer the baseline but also we believe to be more robust, easier to generalize and more directly useful for downstream text recognition tasks. Recent competitions were dominated by deep learning based approaches. The ICDAR2017 competition on BAseline Detection (cBAD2017) [9] was won by the approach proposed by Fink et al. [13], a sliding-window dense prediction using a U-Net architecture [14]. Later, the winning entry was successively surpassed by the ResNet [15] adaptation of Ares Oliveira et al. [16] and by the model proposed by Grüning et al. [17] which added an attention mechanism and developed a sophisticated post-processing step 
based on superpixels. A slightly refined version of the latter also won the cBAD2019 challenge [11]. We use a plain segmentation approach similar to [16] followed by a simple post-processing step designed to work for both text lines and illustrations.

Synthetic data. Training deep networks for both page and text line segmentation requires large amounts of data. For modern documents, Yang et al. [7] and Zhong et al. [8] proposed synthetic document generation engines based on modern formats (respectively Latex and PDF) yielding to large-scale and heterogeneous document datasets. However, these documents are too simple to train a model that perform well on historical documents. To overcome the issue, Capobianco and Marinai [18] as well as Journet et al. [19] proposed toolkits to expand an existing annotated document dataset by generating similar semi-synthetic documents with the help of advanced data augmentation strategies. Resulting datasets are thus limited in diversity and they are not designed for generalization to new datasets. Besides, all the proposed generation processes either don't include graphical elements, or rely on very simple ones. On the contrary, we propose a complete synthetic document generation approach that generalizes well to a large variety of historical document datasets for both text line and illustration segmentation. Note that our synthetic documents can also be used for text recognition, similar to [20].

\section{APPROACH}

We consider line-level page segmentation as a pixel-wise classification task and propose to solve it using a deep neural network trained on our large-scale synthetic document dataset SynDoc, followed by a simple connected component filtering. In this section, we first introduce our synthetic data generation engine, then describe our segmentation method.

\section{A. Synthetic document generation and labeling}

While several datasets [5]-[8] are available for page segmentation, they do not embrace the wide diversity of historical documents. Furthermore, text regions are always annotated as coarse text blocks preventing straightforward line extractions. To address these issues, we created a fast and scalable synthetic document generation engine with pixel-wise annotations and use it to generate a dataset of 10k images called SynDoc. We first present an overview of the generation process, then the basic elements we used to obtain challenging data and finally the labeling we designed for optimal generalization. Examples of generated documents can be seen in Fig. 1.

1) Document generation process: The document generation process includes three randomized steps. First, a page background is selected from a set of 177 empty pages we collected and undergoes augmentations: it can be symmetrized to mimic a double page or pasted on a contextual image picked from a set of 15 images. Second, a grid page layout is drawn and each empty cell is filled with an element with random margins. In the case the element is graphical, a horizontal and vertical caption can be added. Third, different forms of degradations are applied to avoid overfitting and increase robustness: Gaussian blur, structured noise (random shapes) addition and bleed-through. Bleed-through degradation is critical in manuscript layout analysis and we perform it by overlaying another grid of random elements with low opacity. This modular approach enables to easily add new types of elements.

2) Element generation: We implemented four types of elements which we found to be critical to obtain good results on real historical documents:

- text: we used texts scraped from random wikipedia pages. Texts can then be generated in 5 different layouts: caption, floating-word, paragraph, table and title. We augment them using translation (to Arabic or Chinese), font changes (selection from 405 fonts we downloaded from the $w_{e b}^{2}$ and formatting such as size or spacing), justification, strike through, underlining, rotation and bounding box addition,

- image: we used the Wikiart dataset ${ }^{1}$ which contains much more difficult images for page segmentation than natural image datasets.

- drawing: we transformed images scraped from random wikipedia pages into drawings by blending them with their blurred negative through color dodging,

- glyph: we collected 91 decorated fonts from the $\mathrm{web}^{2}$ and a random uppercase letter is picked to generate a glyph.

At generation time, we perform generic on-the-fly augmentations such as blurring, colorization and opacity variation. Each element class is associated to its own labeling described in the next paragraph. The benefits of using background augmentations, drawings, glyphs, text translation and bleedthrough are experimentally demonstrated in Sec. IV-C.

3) Element labeling: Even though labeling can be elementspecific, we argue a wide diversity of labels makes it difficult to generalize to new types of documents. Following most text line detection competitions [9]-[11], we thus label all text elements the same way and associate to all graphical elements a single illustration label.

While labeling images is straightforward, we label the shape of glyphs and drawings using closing operations. Contrary to bounding box or contour labels, we believe such labeling is not only accurate enough to extract the targeted region without surrounding elements but also coarse enough to be easily learned by the model. For text, to perform page segmentation at line level, we adopted $\mathrm{x}$-height representation, which corresponds to the core area of the text without ascenders and descenders. Unlike bounding box or baseline labels, it enables a straightforward line extraction while preventing lines from merging. Besides, we expect that $\mathrm{x}$-height representation has a better generalization power to unknown fonts than baseline labels as it doesn't require to infer text orientation. Because we still observed lines vertically merged by small pixel bridges in the case of thin interline spaces, we labeled border regions

\footnotetext{
${ }^{1}$ http://www.wikiart.org/

${ }^{2}$ https://www.dafont.com/, https://fonts.google.com/
} 

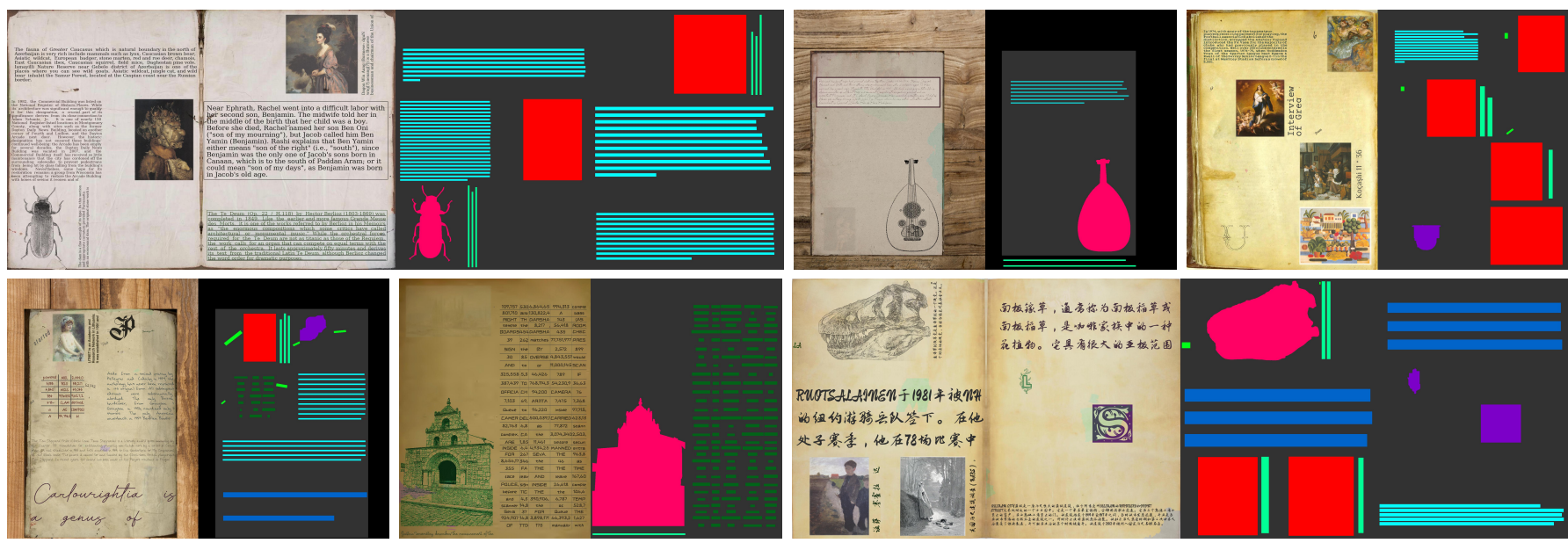

Fig. 1. SynDoc examples with ground-truth. Elements are: page-bkg,

around the text representations to help the model learn interline spaces. We experimentally show in Sec. IV-C the improvements stemming from such text labeling choices.

\section{B. Segmentation method}

We perform line-level page segmentation using a fully convolutional network, optimized with a standard cross-entropy loss and followed by a simple post-processing.

1) Network architecture: Similar to [16], we use a simple encoder-decoder architecture combining the descriptive power of ResNet [15] with the localization recovering capacity of U-Net [14]. Compared to [16], we use a smaller ResNet-18 as backbone encoder since detecting text lines requires keeping document images as large as possible, which constraints memory, and we perform small modifications in the architecture resulting in better performances. The full network architecture is summarized in Fig. 2.

We replaced the max-pooling operation in ResNet conv2 block by a 2 -strided $3 \times 3$ convolutional layer, as max-pooling has been shown [21] to lead to gridding artifacts. The decoder is composed of 5 upscaling blocks and a final convolutional layer which assigns a class to each pixel. Each upscaling block is composed of an upscaled version of the previous feature map concatenated with the corresponding encoding feature map and a 3x3 convolutional layer. Because text lines can be small,

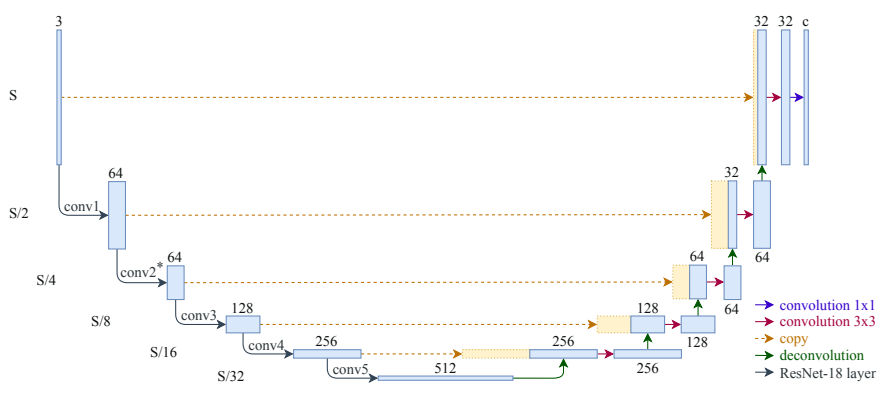

Fig. 2. Network architecture. ${ }^{*}$ indicates max-pooling replacement. we upsample features using deconvolutional [22] layers with stride 2 rather than bilinear interpolation.

2) Post-processing: We use a simple post-processing step filtering out connected components with low area using a class-specific ratio threshold.

To compare with state-of-the-art baseline detection methods, we either retrieve baselines from the segmentation maps, or for low shot comparisons change our labels to directly predict baselines. To compute a baseline from a x-height component, we first fit a straight line to retrieve the text orientation and its bottom line. We then fit to the latter a 5-degree polynomial to get a smooth baseline prediction. This process assumes that the page is well oriented and it particularly fails in the case of transposed texts $\left(90^{\circ}\right.$ or $180^{\circ}$ rotated).

3) Implementation details: All images are resized so that their larger side is 1280 pixels, keeping the aspect ratio constant. We perform per-channel standardization and during training several on-the-fly data augmentations including Gaussian blur, brightness and contrast variation, image rotation and transposition. When fine-tuning on real datasets, we also perform random scaling which we found to be critical for high-performances. We limit the maximum number of pixels to $3.5 \times 10^{6}$ to avoid memory error while scaling. For memory reasons, we process one sample per batch and use Instance Normalization [23] with a momentum of 0.1 instead of batch normalization. We use ImageNet [24] pre-trained weights for the encoder, which significantly speeds up the training, and Xavier initialization [25] for the other convolutional layers. We train for 100 epochs with Adam optimizer [26] with a weight decay of $10^{-6}$. Learning rate is initially set to 0.001 and divided by 2 after 30, 60 and 80 epochs. On a Nvidia GeForce RTX 2080 Ti GPU, training takes approximately 3 days and single image inference takes 1.06 second.

\section{EXPERIMENTS}

In this section, we first introduce the sets of datasets used for text line detection and illustration segmentation evaluations, including our new IlluHisDoc. Then, we present quantitative 

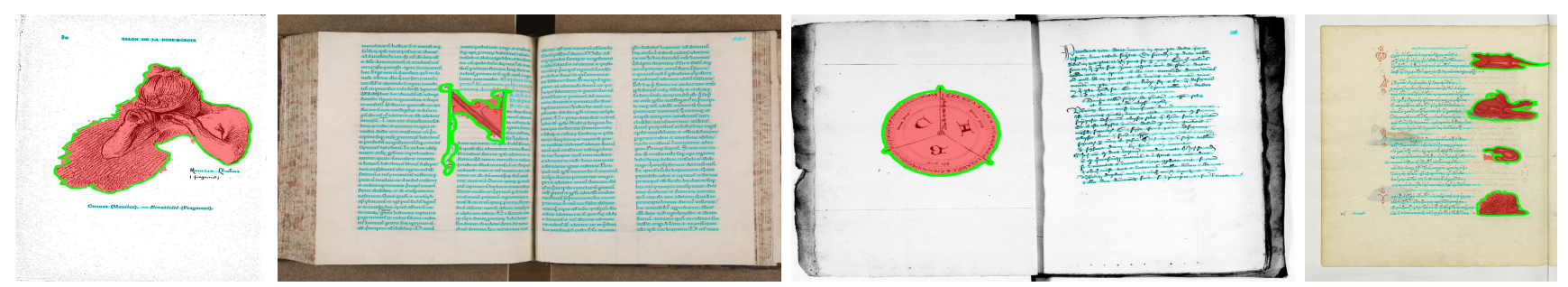

Fig. 3. Examples from IlluHisDoc dataset with ground-truth and segmentation outputs of our method ( illustration and text ). From left to right: printed documents (P), manuscripts with illuminations (MSI), manuscripts with scientific diagrams (MSS), manuscripts with drawings (MSD).

results with comparisons to state-of-the-art of both our off-theshelf and fine-tuned approach. Finally, we present a methodical ablation study of our approach.

\section{A. Datasets}

The cBAD competitions [9], [11] involve large datasets with a great variety of historical document images and are the standard benchmarks for text line detection. To the best of our knowledge, there is no dataset for illustration segmentation with such a diversity. Hence, we evaluate our method using three diverse datasets: Mandragore ${ }^{3}$, RASM2019 ${ }^{4}$ and our proposed IlluHisDoc dataset.

1) $\boldsymbol{c B A D 2 0 1 7}$ and $\boldsymbol{c B A D 2 0 1 9 :}$ Dataset for $\mathrm{CBAD} 2017$ is split in two, Simple and Complex Tracks, with respectively 216 and 270 images for training, 539 and 1010 images for evaluation. Larger and more diversified, cBAD2019 contains 1510 training and 1511 evaluation images.

2) Mandragore: Dedicated to the illustration detection, the dataset is composed of 8 manuscripts, gathering 2807 pages including 631 illustrations annotated with bounding boxes. Because of inconsistent annotations, we removed Français 2692 and Latin 757 manuscripts as well as all book spine and cover images, resulting in a dataset of 1691 images.

3) RASM2019: Dataset is composed of Arabic scientific handwritten manuscripts. Initially meant for text detection and recognition, it also includes annotations for scientific figures labeled as graphics and images, which we merged into a global illustration class. Test set consists in 100 images.

4) IlluHisDoc: To provide a more representative evaluation for illustration segmentation, we created a new test dataset dubbed IlluHisDoc (Illustrated Historical Documents). We designed it to include diverse types of illustrations relevant for digital humanities and to embrace a wide variety of documents, layouts and degradations. Document images were mainly downloaded from Gallica ${ }^{5}$. We explicitly split IlluHisDoc in 4 parts corresponding to different types of illustrations:

- $P: 5$ printed documents that comprise multiple forms of illustration (drawing, ornament, painting, photo),

- MSS: 5 manuscripts with scientific diagrams,

- MSI: 5 manuscripts with illuminations,

- MSD: 5 manuscripts with drawings.

\footnotetext{
${ }^{3} \mathrm{http}: / /$ api.bnf.fr/mandragore-echantillon-segmente-2019

${ }^{4}$ https://www.primaresearch.org/RASM2019/resources

${ }^{5}$ gallica.bnf.fr, Bibliothèque nationale de France
}

TABLE I

RESULTS FOR CBAD2017 DATASET

\begin{tabular}{|c|c|c|c|c|c|c|c|}
\hline \multirow[t]{2}{*}{ Method } & \multirow{2}{*}{$\begin{array}{l}\text { Training } \\
\text { set used }\end{array}$} & \multicolumn{3}{|c|}{ Simple Track } & \multicolumn{3}{|c|}{ Complex Track } \\
\hline & & P-val & R-val & F-val & P-val & R-val & F-val \\
\hline Tesseract 4 & & 0.396 & 0.545 & 0.459 & 0.322 & 0.520 & 0.398 \\
\hline Ours (off-the-shelf) & & 0.871 & 0.930 & 0.900 & 0.844 & 0.782 & 0.812 \\
\hline LITIS [9], [12] & $\checkmark$ & 0.780 & 0.836 & 0.807 & - & - & - \\
\hline IRISA [9] & $\checkmark$ & 0.883 & 0.877 & 0.880 & 0.692 & 0.772 & 0.730 \\
\hline UPVLC [9] & $\checkmark$ & 0.937 & 0.855 & 0.894 & 0.833 & 0.606 & 0.702 \\
\hline BYU [9] & $\checkmark$ & 0.878 & 0.907 & 0.892 & 0.773 & 0.820 & 0.796 \\
\hline dhSegment [16] & $\checkmark$ & 0.88 & 0.97 & 0.92 & 0.79 & 0.95 & 0.86 \\
\hline DMRZ [9], [13] & $\checkmark$ & 0.973 & 0.970 & 0.971 & 0.854 & 0.863 & 0.859 \\
\hline Planet [17] & $\checkmark$ & 0.98 & 0.98 & 0.978 & 0.93 & 0.92 & 0.922 \\
\hline Ours (fine-tuned) & $\checkmark$ & 0.948 & 0.978 & 0.963 & 0.883 & 0.947 & 0.914 \\
\hline
\end{tabular}

TABLE II

RESULTS FOR CBAD2019 DATASET

\begin{tabular}{lcccc}
\hline Method & Training set used & P-val & R-val & F-val \\
\hline Tesseract4 & & 0.442 & 0.552 & 0.491 \\
Ours (off-the-shelf) & & $\mathbf{0 . 8 4 4}$ & $\mathbf{0 . 8 1 5}$ & $\mathbf{0 . 8 2 9}$ \\
\hline Baseline [11] & $\checkmark$ & 0.773 & 0.743 & 0.758 \\
TJNU [11] & $\checkmark$ & 0.852 & 0.885 & 0.868 \\
UPVLC [11] & $\checkmark$ & 0.911 & 0.902 & 0.907 \\
DMRZ [11] & $\checkmark$ & 0.925 & 0.905 & 0.915 \\
Planet [11] & $\checkmark$ & $\mathbf{0 . 9 3 7}$ & 0.926 & $\mathbf{0 . 9 3 1}$ \\
Ours (fine-tuned) & $\checkmark$ & 0.920 & $\mathbf{0 . 9 3 1}$ & 0.925 \\
\hline
\end{tabular}

In each source document, we annotated 10 images with at least one illustration and 10 images without any resulting in 400 pages. Annotations were performed at pixel-level using VGG Image Annotator [27]. Note that the aim of this dataset is to evaluate the generalization capability of out-of-the-box solutions to generalize to unseen data and not for training. Examples of our four types of documents are shown in Fig. 3.

\section{B. Results}

In this section, we evaluate our approach for both baseline detection and illustration segmentation. We compare to the offthe-shelf open-source Tesseract 4 and state-of-the-art methods.

1) Baseline detection: We evaluate our method for baseline detection on the test split of cBAD2017 and cBAD2019 using the competition evaluation scheme. We report results for offthe-shelf and fine-tuned configurations in Table I and II.

While never trained on real data, our off-the-shelf approach provides good results across the three benchmarks, outperforming Tesseract 4 by a large margin and showing results 


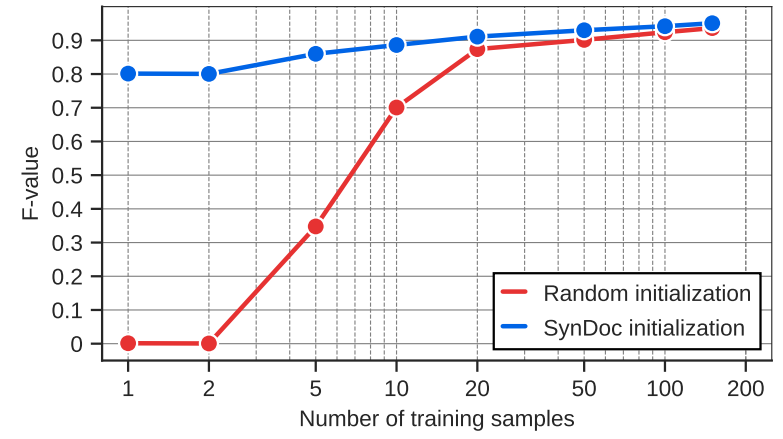

Fig. 4. F-value with different amount of training data on cBAD2017 Simple Track using networks randomly initialized and pre-trained on SynDoc.

comparable to the weaker methods trained on real data. This shows that our method trained only on computer-generated data generalizes well to real and complex handwritten data.

After fine-tuning on the respective training sets, our method leads to results on par with state-of-the-art methods. This is a strong result since these methods typically involve very advanced and specific post-processing steps, while ours is very simple and common across all datasets and element types. Our performance could likely be further improved, for example using the superpixel-based post-processing of [17] which they demonstrate to provide a remarkable boost. This is however orthogonal to the goal of our work, which is providing a simple, robust and generic method.

Our synthetic training data could also be used to initialize a network and fine-tune it with a few real examples. To evaluate this setup, we split cBAD2017 Simple Track training set in two, keeping 40 samples as evaluation set and training on the rest. In Fig. 4, we compare random and SynDoc initializations with an increasing number of training samples to fine-tune the network. Remarkably, SynDoc initialization consistently leads to better results, with particularly large gaps when less than 20 annotated samples are available.

2) Illustration segmentation: We evaluate our off-theshelf approach for illustration segmentation on Mandragore, RASM2019 and IlluHisDoc. Mean Intersection over Union (mIoU) scores are reported in Table III.

To validate the benefits of both our synthetic dataset and our segmentation approach, we also report results using the synthetic PubLayNet dataset introduced in [8] as well as one of their benchmarked methods, Mask-RCNN [28]. Our full method provides results far above any baseline, including Tesseract4 which generalizes poorly to historical documents. Two effects can be clearly identified. First, for both training datasets, our segmentation approach provides much better results than a detection-based approach across all datasets. Second, training on SynDoc provides much better generalization on historical datasets than training on PubLayNet both for Mask-RCNN and our segmentation method.

\section{Ablations}

We here show the benefits of our contributions for synthetic document generation, text labeling and network architecture.
TABLE III

ILLUSTRATION SEGMENTATION (MIOU IN \%)

\begin{tabular}{lcccccccc}
\hline & & & & \multicolumn{5}{c}{ IlluHisDoc } \\
\cline { 5 - 9 } Method & Training & Mandra. & RASM & avg & P & MSS & MSI & MSD \\
\hline Tesseract4 & & 17.2 & 6.0 & 14.8 & 41.4 & 9.2 & 2.0 & 6.5 \\
M-RCNN & PubLay. & 9.8 & 4.2 & 11.5 & 34.3 & 3.1 & 1.5 & 7.2 \\
Ours & PubLay. & 18.3 & 14.8 & 24.3 & 57.7 & 16.9 & 5.3 & 17.2 \\
M-RCNN & SynDoc & 72.3 & 36.9 & 55.4 & 93.5 & 60.3 & 40.3 & 27.6 \\
Ours & SynDoc & $\mathbf{8 6 . 6}$ & $\mathbf{7 1 . 0}$ & $\mathbf{7 6 . 1}$ & $\mathbf{9 7 . 2}$ & $\mathbf{6 1 . 8}$ & $\mathbf{7 6 . 8}$ & $\mathbf{6 8 . 5}$ \\
\hline
\end{tabular}

TABLE IV

ABLATION STUDY ON SYNDOC IMPROVEMENTS (MIOU IN \%)

\begin{tabular}{lccc}
\hline Experiment & Mandragore & RASM2019 & IlluHisDoc \\
\hline SynDoc & $\mathbf{8 6 . 6}$ & $\mathbf{7 1 . 0}$ & 76.1 \\
w/o bleed-through & 84.3 & 67.0 & $\mathbf{7 7 . 2}$ \\
w/o text translation & 84.4 & 63.6 & 76.6 \\
w/o drawing \& glyph & 80.5 & 23.6 & 52.5 \\
w/o bkg augmentations & 55.9 & 44.6 & 44.1 \\
\hline
\end{tabular}

All experiments are trained on SynDoc following Sec. III-B3.

1) SynDoc: In Table IV, we evaluate the improvements proposed for synthetic document generation by systematically removing them from the generation engine. Evaluation is done for illustration segmentation in Mandragore, RASM2019 and IlluHisDoc. Results show that adding bleed-through, texts in different languages, drawings and glyphs as well as augmenting page backgrounds with double pages or contextual images, all contribute to our high performances in amounts that differ depending on the specificity of each test dataset.

2) Text labeling: In Table V, we show the benefits of our $\mathrm{x}$-height representation with border labels for text lines. We train our approach with different labels and evaluate baseline detection on cBAD2017 and cBAD2019. Two main effects can be seen. First, predicting $\mathrm{x}$-height representation and using the prediction to infer baselines performs better than directly predicting the baseline. Second, adding border labels dramatically boosts performances both when training with $\mathrm{x}$ height and baseline representations. Nonetheless, the boost is much clearer when using $\mathrm{x}$-height, because in this case borders are necessary to avoid merging different close lines. On the three benchmarks, the combination of $\mathrm{x}$-height with border labels provides a very significant boost, allowing our method to perform well without advanced post-processing.

3) Network architecture: We now validate the benefits of the architecture changes we made compared to dhSegment [16]: a simple ResNet-18 backbone, the replacement of

TABLE V

ABLATION EXPERIMENTS FOR TEXT LABELING CHOICES (F-VALUE)

\begin{tabular}{lcccc}
\hline & & \multicolumn{2}{c}{ cBAD2017 } & \\
\cline { 3 - 4 } text label & border label & Simple & Complex & cBAD2019 \\
\hline baseline & & 0.663 & 0.719 & 0.637 \\
baseline & $\checkmark$ & 0.714 & 0.771 & 0.678 \\
x-height & & 0.749 & 0.724 & 0.758 \\
x-height & $\checkmark$ & $\mathbf{0 . 9 0 0}$ & $\mathbf{0 . 8 1 2}$ & $\mathbf{0 . 8 2 9}$ \\
\hline
\end{tabular}


TABLE VI

ABLATION EXPERIMENTS FOR ARCHITECTURE MODIFICATIONS EVALUATED ON SYNDOC (IOU IN \%)

\begin{tabular}{cccccccc}
\hline conv2 & upscaling & \#param & bkg & illustration & text & border & avg \\
\hline max-pooling & bilinear & $13.6 \mathrm{M}$ & 97.5 & 94.6 & 85.8 & 74.6 & 88.1 \\
strided conv & bilinear & $13.6 \mathrm{M}$ & 97.6 & $\mathbf{9 5 . 0}$ & 86.6 & 75.5 & 88.7 \\
strided conv & deconv & $14.4 \mathrm{M}$ & $\mathbf{9 7 . 7}$ & 94.9 & $\mathbf{8 7 . 6}$ & $\mathbf{7 7 . 2}$ & $\mathbf{8 9 . 3}$ \\
\hline
\end{tabular}

the max-pooling by a strided convolution and the deconvolutional upscaling. In Table VI, we evaluate three variants of our model on a synthetic testing set, with and without maxpooling and upscaling replacements using IoU for all labels. This enables us to obtain results similar to those of dhSegment on the same data $(88.8 \%$ in average compared to $89.3 \%$ for our architecture, dhSegment being slightly better for illustrations and worse for texts) while using much less parameters (14.4M versus $32.9 \mathrm{M}$ for dhSegment). This is important as we found that high-resolution images and upscaling data augmentation when fine-tuning were crucial to obtain results on par with state-of-the-art baseline detection methods.

\section{CONClusion}

To the best of our knowledge, we presented the first robust off-the-shelf system for generic element extraction in historical documents. Our approach relies on a single network and simple post-processing that simultaneously perform text line and illustration segmentation. Its success is based on two key components we introduced: (i) a rich, fast and modular synthetic document generation engine and (ii) an adapted segmentation network that predicts bounding shapes for illustrations and $\mathrm{x}$-height+border representation for text lines. We demonstrated our off-the-shelf approach provides, without any fine-tuning, remarkable performances across a wide variety of challenging datasets. Furthermore, when annotated training images are available, our network can be used as a good initialization for fine-tuning and leads to results on par with the more complex state-of-the-art approaches.

We see our work as a first step toward the development of universal off-the-shelf open-sourced methods for practical historical document analysis. Indeed, a lot of efforts has been dedicated to boosting performances on specialized challenging datasets. Yet, we believe that generic approaches that do not rely on specific trainings for each type of document and task are also an important challenge and can have a strong impact to increase applications in the humanities. We also think our synthetic generation engine will be easy to improve on by adding new elements and more advanced augmentations for even greater generalization capabilities.

\section{ACKNOWLEDGMENTS}

This work was supported in part by ANR project EnHerit ANR-17-CE23-0008, project Rapid Tabasco and gifts from Adobe. We thank Béatrice Joyeux-Prunel, K. Bender, Joanna Fronska, Matthieu Husson, Stavros Lazaris, Galla Topalian, Claudia Rabel, Jean-Philippe Moreux and Alexandre Turc for their help in the data collection and fruitful discussions.
We also thank François Darmon, Pierre-Guillaume Raverdy, Tristan Dot and Ryad Kaoua for code testing and feedbacks.

\section{REFERENCES}

[1] L. Gao, X. Yi, Z. Jiang, L. Hao, and Z. Tang, "ICDAR2017 Competition on Page Object Detection," in ICDAR, 2017.

[2] F. Simistira, M. Bouillon, M. Seuret, M. Wursch, M. Alberti, R. Ingold, and M. Liwicki, "ICDAR2017 Competition on Layout Analysis for Challenging Medieval Manuscripts," in ICDAR, 2017.

[3] C. Clausner, A. Antonacopoulos, and S. Pletschacher, "ICDAR2019 Competition on Recognition of Documents with Complex Layouts RDCL2019," in ICDAR, 2019.

[4] C. Clausner, A. Antonacopoulos, T. Derrick, and S. Pletschacher, "ICDAR2019 Competition on Recognition of Early Indian Printed Documents - REID2019," in ICDAR, 2019.

[5] A. Antonacopoulos, D. Bridson, C. Papadopoulos, and S. Pletschacher, "A Realistic Dataset for Performance Evaluation of Document Layout Analysis," in ICDAR, 2009.

[6] C. Clausner, C. Papadopoulos, S. Pletschacher, and A. Antonacopoulos, "The ENP image and ground truth dataset of historical newspapers," in ICDAR, 2015.

[7] X. Yang, E. Yumer, P. Asente, M. Kraley, D. Kifer, and C. L. Giles, "Learning to Extract Semantic Structure from Documents Using Multimodal Fully Convolutional Neural Networks," in CVPR, 2017.

[8] X. Zhong, J. Tang, and A. Jimeno Yepes, "PubLayNet: Largest Dataset Ever for Document Layout Analysis," in ICDAR, 2019.

[9] M. Diem, F. Kleber, S. Fiel, T. Gruning, and B. Gatos, "cBAD: ICDAR2017 Competition on Baseline Detection," in ICDAR, 2017.

[10] C. Clausner, A. Antonacopoulos, N. Mcgregor, and D. Wilson-Nunn, "ICFHR 2018 Competition on Recognition of Historical Arabic Scientific Manuscripts - RASM2018," in ICFHR, 2018.

[11] M. Diem, F. Kleber, R. Sablatnig, and B. Gatos, "cBAD: ICDAR2019 Competition on Baseline Detection," in ICDAR, 2019.

[12] G. Renton, C. Chatelain, S. Adam, C. Kermorvant, and T. Paquet, "Handwritten Text Line Segmentation Using Fully Convolutional Network," in ICDAR, 2017.

[13] M. Fink, T. Layer, G. Mackenbrock, and M. Sprinzl, "Baseline Detection in Historical Documents Using Convolutional U-Nets," in DAS, 2018.

[14] O. Ronneberger, P. Fischer, and T. Brox, "U-net: Convolutional networks for biomedical image segmentation," in MICCAI, 2015.

[15] K. He, X. Zhang, S. Ren, and J. Sun, "Deep Residual Learning for Image Recognition," in CVPR, 2016.

[16] S. Ares Oliveira, B. Seguin, and F. Kaplan, "dhSegment: A Generic Deep-Learning Approach for Document Segmentation," in ICFHR, 2018.

[17] T. Grüning, G. Leifert, T. Strauß, J. Michael, and R. Labahn, "A twostage method for text line detection in historical documents," IJDAR, 2019.

[18] S. Capobianco and S. Marinai, "DocEmul: A Toolkit to Generate Structured Historical Documents," in ICDAR, 2017.

[19] N. Journet, M. Visani, B. Mansencal, K. Van-Cuong, and A. Billy, "DocCreator: A New Software for Creating Synthetic Ground-Truthed Document Images," Journal Imaging, vol. 3, p. 62, 2017.

[20] A. Gupta, A. Vedaldi, and A. Zisserman, "Learning to read by spelling: Towards unsupervised text recognition," in ICVGIP, 2018.

[21] F. Yu, V. Koltun, and T. Funkhouser, "Dilated Residual Networks," in CVPR, 2017.

[22] H. Noh, S. Hong, and B. Han, "Learning Deconvolution Network for Semantic Segmentation," in ICCV, 2015.

[23] D. Ulyanov, A. Vedaldi, and V. Lempitsky, "Instance Normalization: The Missing Ingredient for Fast Stylization," arXiv:1607.08022, 2016.

[24] J. Deng, W. Dong, R. Socher, L.-J. Li, Kai Li, and Li Fei-Fei, "ImageNet: A large-scale hierarchical image database," in CVPR, 2009.

[25] X. Glorot and Y. Bengio, "Understanding the difficulty of training deep feedforward neural networks," in AISTATS, 2010.

[26] D. P. Kingma and J. Ba, "Adam: A Method for Stochastic Optimization," in ICLR, 2015.

[27] A. Dutta and A. Zisserman, "The VIA Annotation Software for Images, Audio and Video," ACMMM, 2019.

[28] K. He, G. Gkioxari, P. Dollár, and R. Girshick, "Mask R-CNN," in ICCV, 2017. 